\title{
OPEN Synthesis and design of Ag-Fe bimetallic nanoparticles as antimicrobial synergistic combination therapies against clinically relevant pathogens
}

A. L. Padilla-Cruz ${ }^{1,2}$, J. A. Garza-Cervantes ${ }^{1,2}$, X. G. Vasto-Anzaldo ${ }^{1,2}$, Gerardo García-Rivas ${ }^{3,4}$, A. León-Buitimea ${ }^{1,2} \&$ J. R. Morones-Ramírez ${ }^{1,2}$

The inappropriate use of antibiotics and the inadequate control of infections have led to the emergence of drug-resistant strains. In recent years, metallo-pharmaceutics and metallic nanoparticles have been proposed as potential alternative antimicrobials due to their broad-spectrum antimicrobial properties. Moreover, recent findings have shown that combinations of transition metal compounds can exhibit synergistic antimicrobial properties. Therefore, the synthesis and design of bimetallic nanoparticles is a field worth exploring to harness the interactions between groups of metals and organic complex structures found in different microbial targets, towards the development of more efficient combinatorial antimicrobials composed of synergistic metals. In this study, we present a green synthesis of $\mathrm{Ag}-\mathrm{Fe}$ bimetallic nanoparticles using an aqueous extract from the leaves of Gardenia jasminoides. The characterization of the nanoparticles demonstrated that the synthesis methodology produces homogenously distributed core-shell Ag-Fe structures with spherical shapes and average diameter sizes of $13 \mathrm{~nm}( \pm 6.3 \mathrm{~nm})$. The Ag-Fe bimetallic nanoparticles showed magnetic and antimicrobial properties; the latter were evaluated against six different, clinically relevant multi-drug-resistant microbial strains. The Ag-Fe bimetallic nanoparticles exhibited an antimicrobial (bactericidal) synergistic effect between the two metals composing the bimetallic nanoparticles compared to the effects of the mono-metallic nanoparticles against yeast and both Gram-positive and Gram-negative multidrug-resistant bacteria. Our results provide insight towards the design of bimetallic nanoparticles, synthesized through green chemistry methodologies, to develop synergistic combinatorial antimicrobials with possible applications in both industrial processes and the treatment of infections caused by clinically relevant drug-resistant strains.

The fast spread of drug-resistant infectious diseases poses a global health threat ${ }^{1}$. If the trend continues at the current speed, by 2050, it will lead to 10 million people dying every year from drug-resistant infections ${ }^{2}$. Metal nanoparticles (NPs) have great potential to be utilized as antimicrobial agents ${ }^{3}$. Noble metal NPs, such as silver $\mathrm{Ag})$ and gold $\mathrm{Au}$ ), have been shown to exhibit strong and sustainable antibacterial action against a wide array of microorganisms; therefore, they have been utilized in medical devices, food preservatives, dental resin composites, cosmetics, medical device coatings, implants, and medical instruments ${ }^{4}$. Bimetallic NPs have gained

\footnotetext{
${ }^{1}$ Universidad Autónoma de Nuevo León, UANL, Facultad de Ciencias Químicas, Av. Universidad S/N. CD. Universitaria, San Nicolás de Los Garza, NL 66455, Mexico. ${ }^{2}$ Centro de Investigación en Biotecnología y Nanotecnología, Facultad de Ciencias Químicas, Universidad Autónoma de Nuevo León, Parque de Investigación E Innovación Tecnológica, Km. 10 autopista al Aeropuerto Internacional Mariano Escobedo, Apodaca, Nuevo León 66629, México. ${ }^{3}$ Cátedra de Cardiología Y Medicina Vascular, Escuela de Medicina. Tecnologico de Monterrey, Monterrey, Nuevo León, Mexico. ${ }^{4}$ Centro de Investigación Biomédica, Hospital Zambrano-Hellion, Tecnologico de Monterrey, San Pedro Garza-García, Nuevo León, Mexico. ${ }^{\circledR}$ email: jose.moronesrmr@uanl.edu.mx
} 
specific attention in the last decade due to their optical, electronic, magnetic, and catalytic properties, which, in most cases, are significantly distinct from their monometallic counterparts ${ }^{4}$. Bimetallic NPs are synthesized by combining two different metal elements, resulting in various morphologies and structures ${ }^{5}$.

Green synthesis provides a new scope for NP synthesis since it is an eco-friendly, simple, stable, rapid, and less-costly method ${ }^{6}$. In general, the synthesis of bimetallic NPs involves the mixing of two different aqueous metal solutions with an environmentally friendly reducing agent, such as a plant extract ${ }^{7}$. The plant phytochemicals, with antioxidant or reducing properties, are believed to reduce metal ions into metal nanoparticles ${ }^{8}$. Theoretically, metal ions with a stronger reduction potential are reduced faster than metal ions with a weaker reduction potential. Such is the well-known system of the $\mathrm{Au}-\mathrm{Ag}$ bimetallic NPs, where Au ions are reduced first, forming the nuclei, while Ag ions are reduced later and adsorbed onto the Au particles, forming a core-shell structure ${ }^{9}$.

Gardenia jasminoides (G. jasminoides), an evergreen tree, is commonly used in traditional Chinese medicine due to its multiple biological activities (antioxidant properties, hypoglycemic effect, and inhibition of inflammation $)^{10}$. It has been reported that G. jasminoides leaves possess alkaloid, flavonoid, saponin, tannin, and phenolic compounds ${ }^{11}$. Therefore, extracts from the leaves of $G$. jasminoides possess a high reducing potential for the synthesis of metallic nanoparticles such as palladium, iron, and silver ${ }^{12-14}$.

The synthesis of bimetallic nanoparticles using plant extracts is a promising method since it has proved to be cheaper, safer, simpler, quicker, and easier than conventional methods ${ }^{8}$. The green synthesis of $\mathrm{Au}-\mathrm{Ag}$ bimetallic nanoparticles and their biomedical applications have been extensively reported ${ }^{15-18}$. On the other hand, silver-iron (Ag-Fe) bimetallic nanoparticles have several applications in optical, medical, and the remediation fields ${ }^{19-22}$. However, there are synthesis methods and important properties and characteristics that still need to be investigated.

Therefore, in this study we synthesized Ag, Fe, and Ag-Fe bimetallic nanoparticles using G. jasminoides extract as a reducing agent in the redox synthesis of the nanomaterials. We characterized the nanostructures by spectroscopic and microscopic analyses and studied their magnetic properties as well as the minimal inhibitory concentration (MIC) and the minimum bactericidal and fungicidal concentration (MBC and MFC) against clinically relevant pathogenic strains. The findings and results presented in this work contribute to the advancement of knowledge on synthesis and antimicrobial properties of mono and bimetallic nanoparticles composed of $\mathrm{Ag}$ and Fe. We demonstrated the ability to use green synthesis methods to design and synthesize magnetic bimetallic nanoparticles composed of $\mathrm{Ag}$ and $\mathrm{Fe}$ and demonstrated that they exhibit a synergistic antimicrobial effect. Moreover, the results here described give insight towards the development of novel and more effective antimicrobial therapies for different applications and in the possible treatment of infections caused by both sensitive and drug-resistant clinically relevant pathogens.

\section{Results and discussion}

Nanoparticles characterization. Nanomaterials, including bimetallic nanoparticles, are of growing interest in many applications. Bimetallic nanoparticles can exhibit a variety of properties due to the synergistic effect created when two different metals are combined. This phenomena can enhance their features and properties and therefore expand or accentuate their applications as antimicrobial agents, drug delivery systems, and as imaging agents ${ }^{23}$. Nonetheless, while much research has been performed on monometallic nanoparticles, fewer studies have explored the use of bimetallic nanoparticles in those fields. We therefore first explored the synthesis of $\mathrm{Ag}, \mathrm{Fe}$, and Ag-Fe bimetallic NPs by monitoring the color changes in the reaction mixture, followed by their physical-chemical characterization and their antimicrobial properties using various analytical techniques.

For the synthesized Ag-NPs, a wide absorption band can be observed between 380 and $580 \mathrm{~nm}$ with a maximum absorption peak at $425 \mathrm{~nm}$ (Fig. 1). This result agrees with previous reports where the peak corresponding to silver nanoparticles is well documented to be between 390 and $580 \mathrm{~nm}^{24}$. For the case of the Fe-NPs and the synthesis of Ag-Fe bimetallic nanoparticles, both showed the characteristic UV-Vis spectrum, with a peak at $290 \mathrm{~nm}$ and a slight peak at $350 \mathrm{~nm}$, corresponding to metallic iron (Fig. 1). These peaks are related to Fe residues and the Fe surface plasmons' collective oscillation. These absorption bands of surface resonance plasmon have been reported to be present in iron nanoparticles ${ }^{25}$. As can be seen, a characteristic UV-Vis absorption spectrum was displayed when the silver and iron nanoparticles were analyzed independently. However, for the bimetallic nanoparticles, an absorption spectrum resembling that of iron nanoparticles was observed, suggesting the formation of silver-iron core-shell bimetallic nanoparticles. Similar results have been observed in the synthesis of diverse bimetallic nanoparticles ${ }^{26,27}$.

To identify the biomolecules contained in the G. jasminoides leaves extract, responsible for the reduction of $\mathrm{Ag}^{+}$and $\mathrm{Fe}^{+}$ions in the synthesis of nanoparticles, an FT-IR analysis was performed (Fig. 2A). We identified IR bands centered at $3400,2920,1640,1430$, and $1080 \mathrm{~cm}^{-1}$. The band at the $3600-3000 \mathrm{~cm}-1$ region corresponds to hydroxy groups stretching $(\mathrm{O}-\mathrm{H})$. The band at $2950 \mathrm{~cm}^{-1}$ corresponds to the stretching of aliphatic $(\mathrm{C}-\mathrm{H})$. The bands at the 1640 and $1430 \mathrm{~cm}^{-1}$ region can be assigned to $\mathrm{C}=\mathrm{O}$ and $\mathrm{C}=\mathrm{C}$ stretching, respectively. Finally, the $1080 \mathrm{~cm}-1$ band appears due to the stretching of C-O. The FT-IR spectra of the G. jasminoides extract resembled those previously reported ${ }^{12}$. After the synthesis of the Ag, Fe, and Ag-Fe bimetallic nanoparticles with the extract, the absorption peaks appeared weaker than the peaks previous to the reaction synthesis. This result indicates that the extract coated the nanoparticles, reduced metallic ions, and stabilized the nanoparticles during the synthesis. According to several authors, these reducing and stabilizing properties can be attributed to polyphenolic compounds like flavonoids and phenolic acids present in the G. jasminoides extract ${ }^{10,13,28}$. In addition, the RAMAN spectra in Fig. 2B confirmed the presence of two absorption peaks between 1300 and $1500 \mathrm{~cm}^{-1}$, which are characteristic to aromatic rings related to polyphenolic compounds ${ }^{29}$.

The composition, particle size, and size distribution of the Ag-Fe bimetallic NPs were studied by several methods. TEM analysis (Fig. 3A) confirmed that the bimetallic nanoparticles displayed a spherical morphology, 


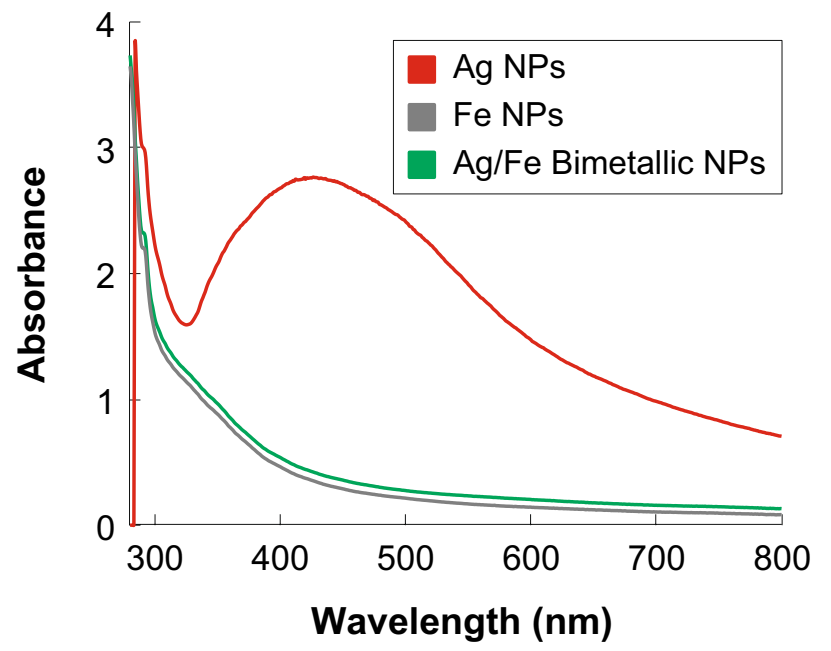

Figure 1. UV-visible absorbance spectra of silver (Ag), iron (Fe) nanoparticles, and Ag-Fe bimetallic nanoparticles synthesized using an aqueous extract from leaves of Gardenia jasminoides.

A

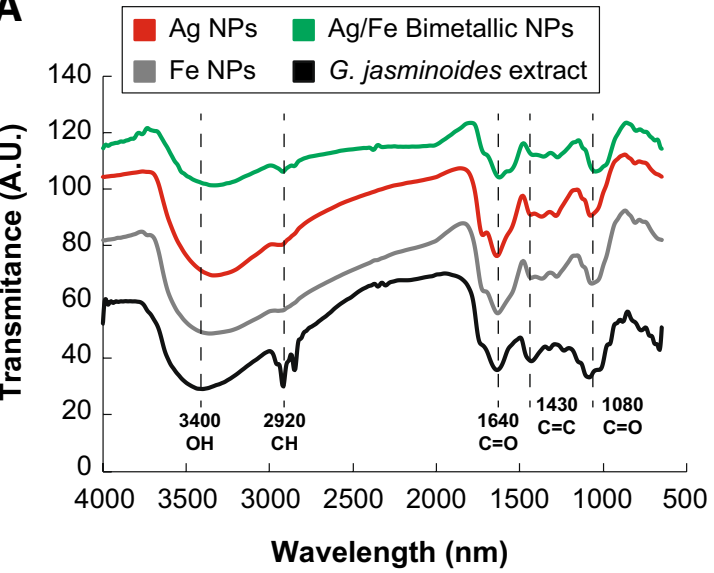

B

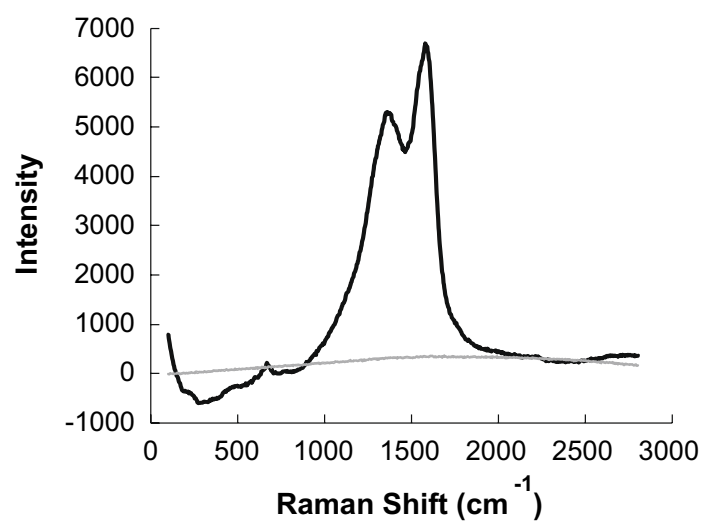

Figure 2. Fourier Transform Infrared (FT-IR) spectra (A) of silver (Ag), iron (Fe) nanoparticles, and Ag-Fe bimetallic nanoparticles synthesized using an aqueous extract from leaves of Gardenia jasminoides and the RAMAN spectra of the (B) aqueous extract from leaves of Gardenia jasminoides.

a homogenous distribution with particles that ranged from 3 to $30 \mathrm{~nm}$ in diameter and an average diameter size of $13 \mathrm{~nm}$ with an SD $+/-6.13 \mathrm{~nm}$ (Fig. 3B and C). The Ag-Fe bimetallic nanoparticles obtained in this study were smaller than those previously reported, where their average size ranged from 20 to $60 \mathrm{~nm}^{30,31}$. However, the differences are related to the reducing and stabilizing agents used during the synthesis process ${ }^{32}$. The highresolution TEM image displayed in Fig. 3B shows clear fringes and morphology that suggests the formation of a core-shell (Ag-core Fe-shell) nano-arrangement of the bimetallic nanoparticles, as reported on the literature ${ }^{30,33}$.

The presence of silver and iron as components of the Ag-Fe bimetallic nanoparticles were confirmed through a dark-field microscopy analysis (Fig. 4A-F). The results showed a clear overlap of the Fe and Ag within the selected, analyzed field, corroborating the bimetallic nanoparticle formation. In addition, carbon (C) was also analyzed to detect the element present in the reducing organic extract. Further analysis using energy dispersive $\mathrm{X}$-Ray (EDX) spectroscopy was carried out to obtain an elemental spectrum in the Ag-Fe bimetallic nanoparticle sample. Figure 4G shows the EDX spectra of the synthesized Ag-Fe bimetallic NPs, where the binding energies of metallic silver are observed, at approximately $3 \mathrm{keV}$, while the peaks around 1,6 , and $7 \mathrm{keV}$ are related to the binding energies of $\mathrm{Fe}^{34,35}$. The elemental composition of the bimetallic nanoparticles was determined by EDX and confirmed that the Ag/Fe bimetallic NPs were composed of $58.59 \% \mathrm{Ag}$ and $41.4 \% \mathrm{Fe}$.

Magnetic properties. Magnetic NPs have been reported as non-immunogenic nanomaterials with excellent biocompatibility, suitable for medical applications ${ }^{36}$. Therefore, the magnetic bimetallic nanoparticles are an outstanding option for diagnostic (sensing and imaging) and therapeutic applications (thermal treatments and drug-delivery systems) ${ }^{37}$. Hence, in the present study, we evaluated the magnetic properties of the Ag-Fe bimetallic NPs (Fig. 5). As can be observed, when an external magnetic field is applied to the Ag-Fe bimetallic NPs, 

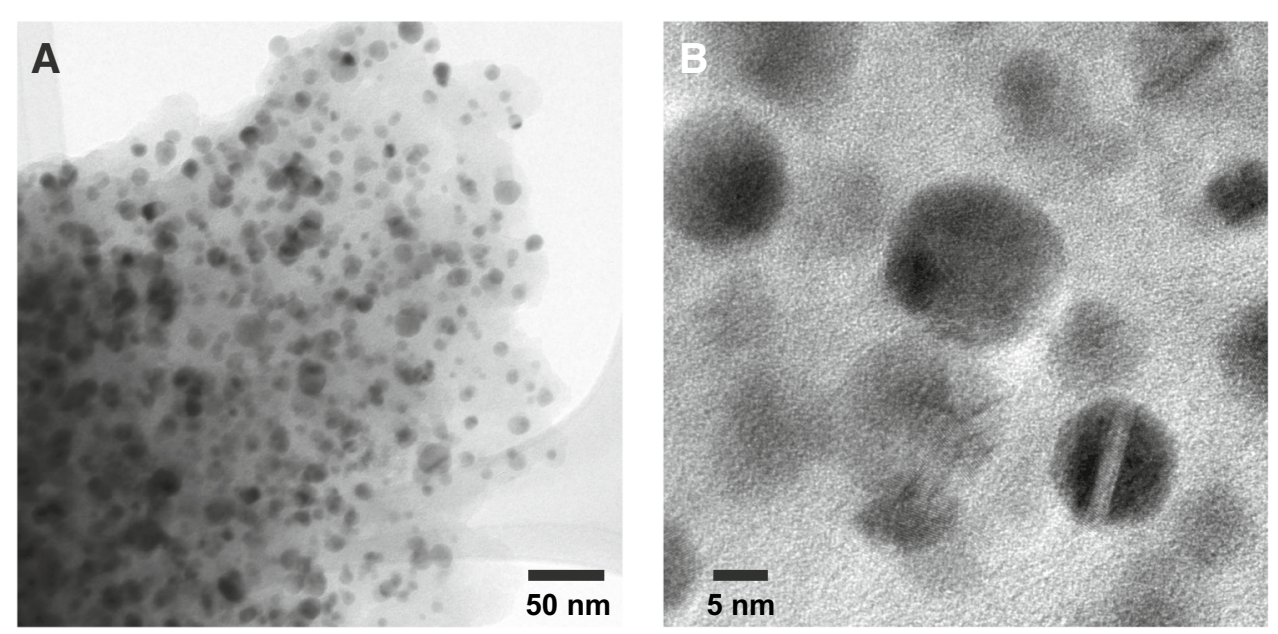

C

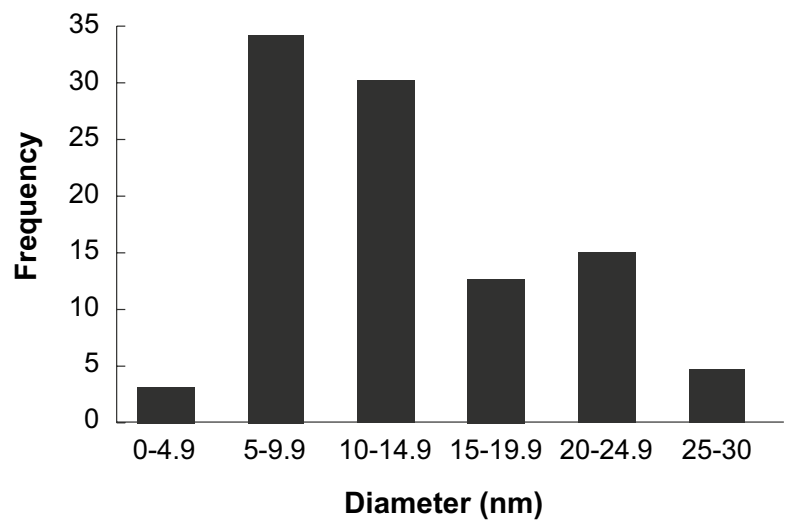

Figure 3. Transmission Electron Microscopy (TEM) characterization of Ag-Fe bimetallic nanoparticles synthesized using an aqueous extract from leaves of Gardenia jasminoides (A) Low magnitude TEM (B) Highresolution TEM images and (C) Size distribution histogram of Ag-Fe bimetallic nanoparticles.

they adhered to the magnet, therefore exhibiting magnetic properties. This magnetic property has previously been described in nanoparticles containing iron ${ }^{30,31}$.

Antimicrobial activity. The emerging resistance in bacteria and the high costs of advanced antimicrobial drugs have encouraged scientists to search for effective, economically viable, and broadly applicable drugs ${ }^{38}$. In search of a new therapeutic alternative, we determined the minimal inhibitory concentrations (MIC) of AgNPs, FeNPs, and Ag-Fe bimetallic NPs against Gram-positive, Gram-negative bacteria, and a yeast using the broth microdilution method (Table 1).

The present study showed that AgNPs had an antimicrobial effect in concentrations ranging from 15.62 to125 $\mathrm{ppm}$. The MIC values of Staphylococcus aureus (S. aureus), a Gram-positive bacteria, were the same in the ATCC and the multidrug-resistant strains $(125 \mathrm{ppm})$. In contrast, Pseudomonas aeruginosa (P. aeruginosa), a Gramnegative bacteria, exhibited lower MIC values (from 15.62 to $62.5 \mathrm{ppm}$ ) in both ATCC and multidrug-resistant strains, compared to the Gram-positive bacteria. Escherichia coli (E. coli; Gram-negative bacteria) and Candida albicans (C. albicans; yeast), displayed MIC values equal to the Gram-positive bacteria, S. aureus (125 ppm). These trends in MIC results are in agreement with previous studies ${ }^{39-41}$.

The MIC results of the present study demonstrated that the FeNPs exhibited no antimicrobial activity $(>250 \mathrm{ppm}$ ), at the explored ranges, in all tested strains. As we previously mentioned, the antibacterial activity of FeNPs has not been thoroughly studied. Most of the studies have evaluated this activity by the well-diffusion method in Gram-positive and Gram-negative bacteria ${ }^{13,42,43}$. We studied the antimicrobial activity using the broth microdilution method; therefore, the results are difficult to comparable.

On the other hand, the MIC values of Ag-Fe bimetallic NPs were similar or lower than those obtained with AgNPs and FeNPs alone. We considered the elemental composition of Ag and $\mathrm{Fe}$ in the bimetallic NPs to analyze their antimicrobial activity ( $58.59 \%$ of $\mathrm{Ag}$ and $41.4 \%$ of $\mathrm{Fe}$ ). In general, the MIC values for $\mathrm{Ag}-\mathrm{Fe}$ bimetallic NPs in S. aureus (S. aureus ATCC 6538, S. aureus ATCC 29213) were comparable to those obtained with AgNPs $(125 \mathrm{ppm})$. However, the elemental composition demonstrated that silver and iron concentrations were 73.23 and $51.75 \mathrm{ppm}$, respectively. Interestingly, the antibacterial effect of $\mathrm{Ag}-\mathrm{Fe}$ bimetallic NPs was more evident against 
A

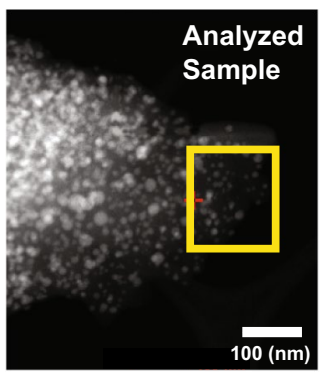

B

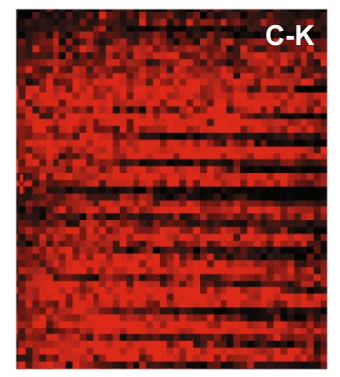

D

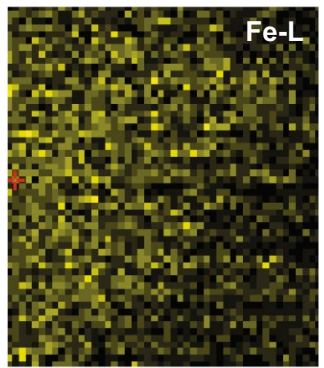

E

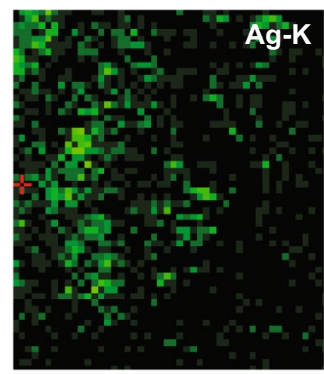

G

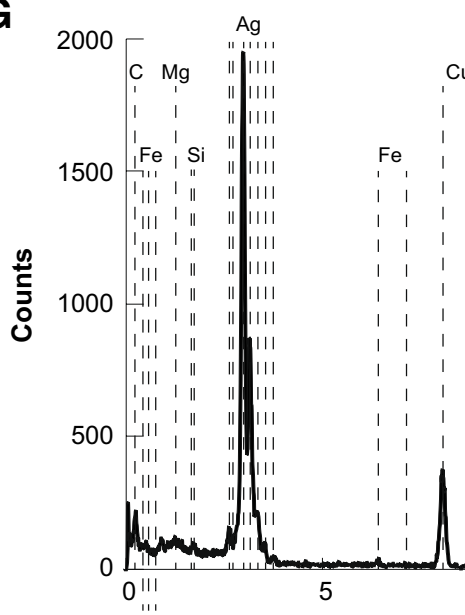

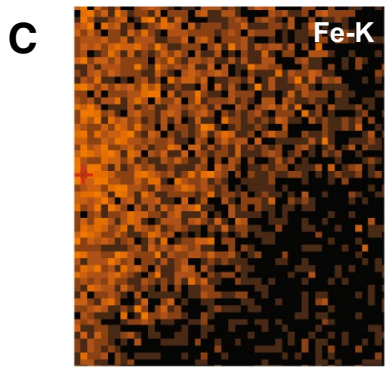

$\mathbf{F}$

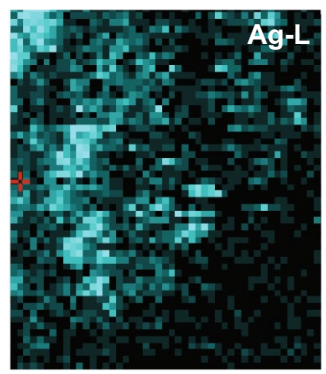

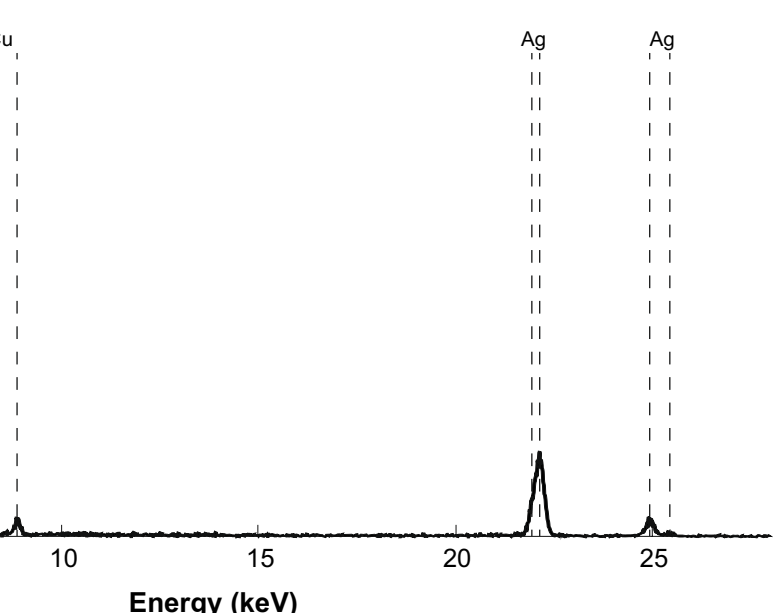

Figure 4. Darkfield image (A)-(F) and energy dispersive X-Ray (EDX) spectra (G) of Ag-Fe bimetallic nanoparticles synthesized using an aqueous extract from leaves of Gardenia jasminoides.

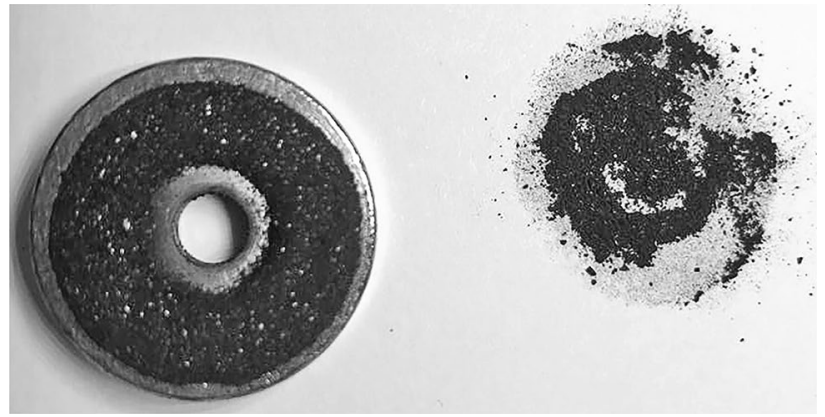

Figure 5. Magnetic property of Ag-Fe bimetallic nanoparticles synthesized using an aqueous extract from leaves of Gardenia jasminoides. 


\begin{tabular}{|c|c|c|c|c|c|c|}
\hline Strain & MIC AgNPs (ppm) & MIC FeNPs (ppm) & $\begin{array}{l}\text { MIC Bimetallic } \\
\text { NPs } \\
\left(\mathrm{Ag} / \mathrm{Fe}^{\star}\right)(\mathrm{ppm})\end{array}$ & $\begin{array}{l}\text { MIC } \\
\mathrm{AgNPs}+\mathrm{FeNPs} \\
\left(\mathrm{Ag} / \mathrm{Fe}^{*}\right)(\mathrm{ppm})\end{array}$ & S value & $\begin{array}{l}\text { MBC or MFC of } \\
\text { Bimetallic NPs } \\
\text { (ppm) }\end{array}$ \\
\hline $\begin{array}{l}\text { S. aureus } \\
\text { ATCC } 6538\end{array}$ & 125 & $>250$ & $125(73.23 / 51.75)$ & $125(73.23 / 51.75)$ & 0.53 & 250 \\
\hline $\begin{array}{l}\text { S. aureus } \\
\text { ATCC } 29,213\end{array}$ & 125 & $>250$ & $125(73.23 / 51.75)$ & $125(73.23 / 51.75)$ & 0.40 & 250 \\
\hline $\begin{array}{l}\text { S. aureus } \\
\text { Multidrug-resistant }\end{array}$ & 125 & $>250$ & $250(146 / 103.5)$ & $250(146 / 103.5)$ & 0.80 & 500 \\
\hline $\begin{array}{l}P \text { aeruginosa } \\
\text { ATCC } 27,853\end{array}$ & 62.5 & $>250$ & $31.25(18.3 / 12.9)$ & $31.25(18.3 / 12.9)$ & 0.89 & 62.5 \\
\hline $\begin{array}{l}P . \text { aeruginosa } \\
\text { ATCC DMS 50,071 }\end{array}$ & 15.62 & $>250$ & $31.25(18.3 / 12.9)$ & $31.25(18.3 / 12.9)$ & 0.80 & 62.5 \\
\hline $\begin{array}{l}\text { P. aeruginosa } \\
\text { Multidrug-resistant }\end{array}$ & 31.25 & $>250$ & $15.62(9.15 / 6.42)$ & $15.62(9.15 / 6.42)$ & 0.90 & 31.25 \\
\hline $\begin{array}{l}\text { E. coli } \\
\text { ATCC } 11,229\end{array}$ & 125 & $>250$ & $125(73.23 / 51.75)$ & $62.5(36.6 / 25.9)$ & 0.40 & 250 \\
\hline $\begin{array}{l}\text { C. albicans } \\
\text { Clinical isolate }\end{array}$ & 125 & $>250$ & $62.5(36.6 / 25.9)$ & $62.5(36.6 / 25.9)$ & 0.08 & 125 \\
\hline
\end{tabular}

Table 1. Minimal Inhibitory Concentration (MIC), S value, Minimum Bactericidal Concentration (MBC) and Minimum Fungicidal Concentration (MFC) of silver nanoparticles (AgNPs), iron nanoparticles (FeNPs), Ag-Fe bimetallic nanoparticles, and combination of AgNPs + FeNPs, prepared using Gardenia jasminoides leaves aqueous extract against clinically relevant pathogens. ${ }^{\star}$ The elemental composition of Ag and $\mathrm{Fe}$ was considered to analyze nanoparticles' antimicrobial activity (bimetallic and AgNPs + FeNPs): $58.59 \%$ of Ag and $41.4 \%$ of Fe. $S$ value: $S>0$ Synergistic; $S=0$ Additive; $S<0$ Antagonistic.

P. aeruginosa. As shown in Table 1, the MIC value in P. aeruginosa ATCC 27853 and ATCC DMS 50071 was $31.25 \mathrm{ppm}$, while the multidrug-resistant strain was $15.62 \mathrm{ppm}$. When we analyzed the elemental composition, it was observed that the concentration of silver and iron was reduced by 3.4 and 19-fold, respectively, compared to AgNPs and FeNPs MICs values. These data show the potential effects of Ag-Fe bimetallic NPs in multidrugresistant bacteria and suggests the importance of the synergistic interactions of these bimetallic NPs, especially for other Gram-negative bacteria with different antibiotic resistance profiles.

Regarding the antifungal activity of Ag-Fe bimetallic NPs in C. albicans (clinical isolate), we observed a MIC value of $62.5 \mathrm{ppm}$ (36.6 and $25.9 \mathrm{ppm}, \mathrm{Ag}$ and Fe, respectively), which was reduced by 3.4 and 9.6-fold, compared to AgNPs and FeNPs MICs values. Moreover, it is worth highlighting that the MICs reported in this study were found to be lower than those previously reported for Ag-Fe bimetallic NPs in S. aureus ATCC, E. coli ATCC, $P$. aeruginosa ATCC, and multidrug-resistant $P$. aeruginos $a^{44,45}$.

The synergistic antimicrobial activity of Ag-Fe bimetallic NPs in a complex matrix, such as bacterial cultures, could follow a non-linear dosage-response behavior. This kind of response is better analyzed when using a synergy test (such as the Bliss independence model) that takes into account the effect of the antimicrobial agent on the bacterial culture, rather than tests that assume all types of antimicrobial agents and their combinations have a linear dosage-respond curve, like the isobologram and the FIC index interpretations ${ }^{46,47}$. We therefore evaluated whether the synergistic effect, measured by the Bliss Independence Model, observed in Ag-Fe bimetallic nanoparticles was conserved or modified after the combination of individually synthesized AgNPs and FeNPs. The results demonstrate that all of the interactions in the bimetallic nanoparticles were found to be synergistic (Table 1). Moreover, the combination of AgNPs + FeNPs was also found to be synergistic in the antimicrobial efficacy against $E$. coli ATCC 11229. In addition, it is critical to keep in mind that one of the main advantages of using bimetallic nanoparticles instead of single metal nanoparticles is that incorporating two individual metals in a single entity makes their synthesis a greener procedure and their delivery a much simpler process ${ }^{15}$. Moreover, this process significantly reduces the quantities of hazardous wastes produced during the biosynthesis of nanoparticles ${ }^{48}$.

The antimicrobial action of Ag-Fe bimetallic NPs seems to depend on the differences in the cell walls or cell membrane composition of the microorganism. However, the antimicrobial mechanism of Ag-Fe bimetallic nanoparticles has not been elucidated yet. Nonetheless, core-shell nanostructures display a more significant effect than that observed in monometallic nanoparticles ${ }^{44}$. Porins could act as a transporter channel for the nanoparticles to and from the cell ${ }^{49}$. It is also known that silver nanoparticles can anchor to the bacterial cell wall and penetrate it. Once inside, they contribute to the formation of free radicals generating intracellular oxidative stress, which leads to cell death ${ }^{50}$. Recently, it has been described that iron might interact with specific amino acids (-SH groups of cysteine) present in proteins of the bacteria cell wall. Particularly, cysteine's thiol side chain has been identified as the most susceptible to gain electrons from the oxidizing species ${ }^{21}$. Therefore, we propose that the antimicrobial mechanism of Ag-Fe bimetallic nanoparticles is carried out in two steps: first, iron, which is coating the nanoparticles, oxidizes the thiol side chain in cysteine leading to changes in the primary structure of proteins, alteration in bacterial cell wall permeability and finally cell death. Second, due to the altered permeability of the cell wall, many nanoparticles are uptake by the bacteria. Silver ions are then released from NPs to the cytoplasm inducing oxidative stress, causing DNA alterations and disruption of membrane morphology. 
Thus, silver and iron contribute to disrupting cellular structures, intracellular biological functions, and lead cell death. Nonetheless, further studies are needed to support this hypothesis.

Next, we determined the minimum bactericidal and fungicidal concentration of Ag-Fe bimetallic NPs to demonstrate their ability to kill bacteria and yeast (Table 1). A bactericidal agent is defined as a material with a ratio of MBC to MIC $\leq 4$. Antibiotics with a ratio of MBC to MIC $>4$ are defined as bacteriostatic agents ${ }^{51}$. The bactericidal agent kills bacteria rapidly and reduces bacterial resistance development; hence they are a better choice for clinicians than bacteriostatic agents ${ }^{52}$. Our results showed that the MBC and MFC values were twice the MIC concentrations; thus, the Ag-Fe bimetallic nanoparticles were bactericidal and fungicidal.

Finally, we conducted a bibliographic search on the evaluation of the biocompatibility of the Ag-Fe bimetallic nanoparticles. A recent study demonstrated that hybrids silver-iron nanoparticles significantly enhanced the cytotoxicity of MCF-7 breast cancer cells. Therefore, these nanoparticles could be a potent anticancer drug ${ }^{53}$. Another study evaluated magnetite/silver nanoparticles biocompatibility in Vero cells (Kidney epithelial cells). The results evidenced low cytotoxicity, hemolytic, and platelet aggregation tendency, which confirm a high biocompatibility ${ }^{54}$. Moreover, a cytotoxicity study of iron-silver core-shell nanoparticles (FeO/AgNPs) was carried out on Vero cell line, and the results showed that these nanoparticles were biocompatible up to $500 \mu \mathrm{gml}^{-1}$ concentration. Hence, even though further studies are required on our specific Ag-Fe bimetallic nanoparticles system, these studies suggest the biocompatibility of our Ag-Fe bimetallic nanoparticles and hint for their potential as biocompatible nanomaterials with biological and biomedical applications.

\section{Conclusions}

The green synthesis of bimetallic nanoparticles using natural extracts is gaining research attention for environmental and medical applications. In the present study, silver nanoparticles (AgNPs), iron nanoparticles (FeNPs), $\mathrm{Ag}-\mathrm{Fe}$ bimetallic nanoparticles, and the combination of AgNPs + FeNPs, were prepared in a redox reaction using the aqueous G. jasminoides leaves extract as a reducing agent. The evaluation of the antimicrobial activity of bimetallic NPs showed synergistic bactericidal and fungicidal effects against Gram-positive and Gram-negative bacteria and yeast. Our results demonstrate the ability to use green synthesis methods to design and synthesize magnetic bimetallic nanoparticles composed of metals that synergize their antimicrobial effects. Moreover, this work gives insight into the development of novel and more effective antimicrobial agents with potential as bactericidal and fungicidal agents, to treat drug-resistant infections, and in other biomedical applications.

\section{Methods}

Microbial strains and culture conditions. Strains used in this study include S. aureus ATCC 6538, S. aureus ATCC 29213, E. coli ATCC 11229, P. aeruginosa ATCC 27853, and P. aeruginosa DSM 50071. Meanwhile, clinical isolates from a Gram-negative multidrug-resistant strain of $P$. aeruginosa (kanamycin, chloramphenicol, and ciprofloxacin-resistant strain), Gram-positive multidrug-resistant strain of S. aureus (kanamycin, chloramphenicol, ciprofloxacin, ampicillin-resistant strain), and a yeast, C. albicans, were obtained from the San Vicente Hospital in Monterrey, Nuevo León, México. All the bacterial assays were performed in Müller-Hinton (MH) media (BD, Bioxon), and all the assays using C. albicans were performed in Yeast Malt (YM) media (BD, Bioxon).

Gardenia jasminoides extract preparation. Leaves from the plant G. jasminoides were collected in Monterrey, Mexico. A voucher specimen (number 634) was deposited in ANSM Herbarium (Universidad Autónoma Agraria Antonio Narro) in Saltillo, Coahuila, México. Leaves were washed using deionized water to remove dust and were dried at room temperature $\left(22-26^{\circ} \mathrm{C}\right)$ for $24 \mathrm{~h}$ before being powdered. An aqueous extract was prepared as follows: two grams of powdered leaves were mixed with $60 \mathrm{~mL}$ of deionized water at room temperature, stirred for $2 \mathrm{~h}$, and filtered immediately through Whatman filter paper. The aqueous extract of G. jasminoides was stored at $4{ }^{\circ} \mathrm{C}$ until their use in the synthesis of NPs.

Synthesis of monometallic nanoparticles. A total of $12.5 \mathrm{~mL}$ of $0.02 \mathrm{M} \mathrm{AgNO}$ were mixed with $12.5 \mathrm{~mL}$ of G. jasminoides extract and heated for $1 \mathrm{~h}$ at $80^{\circ} \mathrm{C}$. The formation of silver nanoparticles was monitored through a change of color of the mixture (from transparent to brownish). The reaction was cooled and centrifuged at 14,000 rpm for $5 \mathrm{~min}$. The product was then washed with $70 \%$ ethanol and lyophilized (FreeZone 6, Labconco, Kansas City, MO, USA) for $24 \mathrm{~h}$ to obtain the purified AgNPs. We followed the previous procedure for the synthesis of the iron nanoparticles; only $12.5 \mathrm{~mL}$ of $0.01 \mathrm{M} \mathrm{Fe}\left(\mathrm{NO}_{3}\right)_{3}$ solution was used instead of the silver solution, and the color change observed was from transparent to black.

Synthesis of Ag-Fe bimetallic nanoparticles. The synthesis of Ag-Fe bimetallic nanoparticles was performed by adding $12.5 \mathrm{~mL}$ of $0.02 \mathrm{M} \mathrm{AgNO}_{3}$ and $12.5 \mathrm{~mL}$ of $0.01 \mathrm{M} \mathrm{Fe}\left(\mathrm{NO}_{3}\right)_{3}$ in a Falcon tube. Then, the tube was mixed and heated until reaching $60^{\circ} \mathrm{C}$. When this temperature was reached, $25 \mathrm{~mL}$ of the filtered G. jasminoides extract was added to the mixture (2:1:1 proportion), kept at constant stir, and heated at $80^{\circ} \mathrm{C}$ for $1 \mathrm{~h}$. The formation of the nanoparticles was monitored and confirmed by a change of color of the mix that changed from transparent to brown (for silver) or black (for iron). The reaction was cooled at room temperature, centrifuged at 14,000 rpm for $5 \mathrm{~min}$, and the supernatant was discharged. The product was then washed with $70 \%$ ethanol, left to dry at room temperature for $24 \mathrm{~h}$, and finally lyophilized (FreeZone 6, Labconco, Kansas City, MO, USA) for another $24 \mathrm{~h}$.

Nanoparticle characterization. Several techniques were used to characterize the synthesized nanoparticles, such as UV-visible (UV-vis) spectra, Fourier-transform infrared (FT-IR) spectroscopy, RAMAN spec- 
troscopy, Transmission electron microscopy (TEM), High Resolution (HR)-TEM, dark-field microscopy, and Energy dispersive X-ray (EDX) analysis. UV-vis absorption spectra of the nanoparticles $(1 \mathrm{mg} / \mathrm{mL}$ in distilled water as a dispersive medium) were studied in the 200-600 nm wavelength range using a Multiskan GO Microplate Spectrophotometer (Thermo Fisher Scientific, USA). FT-IR analysis was performed in the $650-4000 \mathrm{~cm}^{-1}$ range in a Shimadzu IRAffinity-1 spectrometer (Shimadzu, Japan) to determine the plant extract components and the synthesized nanoparticles. The lyophilized NPs and extract $(1 \mathrm{mg} / \mathrm{mL})$ were diluted in $\mathrm{KBr}$ and analyzed by the attenuated total reflectance method. To obtain information about the interactions between the nanoparticles and the plant extract, a RAMAN spectrum of the nanoparticles and the extract was measured using a DXR RAMAN microscope (Thermo Fisher Scientific, USA). The elemental, morphological, and size of the nanoparticles were obtained through a FEI-TITAN $80-300 \mathrm{kV}$ scanning transmission electron microscope. Briefly, the sample was prepared by depositing and evaporating a drop of NPs solution $(1 \mathrm{mg} / \mathrm{mL})$ onto lacey carbon-coated copper grids. The elements presented in NPs were determined by an energy-dispersive spectrometry analyzer integrated in the transmission electron microscope. To analyze the particle size and size distribution, we used the TEM and HR-TEM micrographs to measure de diameter of NPs and then processed them with ImageJ software developed by the National Institutes of Health.

Magnetic properties. A round-shaped magnet was used to produce an external magnetic field, having direct contact with the nanoparticles. If the nanoparticles adhered to the magnet, they were considered to exhibit magnetic properties.

Determination of minimal inhibitory concentration (MIC). The determination of MICs was performed by the microdilution method. Stocks of NPs (mono- and bimetallic) were prepared at a final concentration of $1000 \mathrm{ppm}$ in LB or YM broth. To perform the MIC test, we used a 96-wells microtiter plaque (Corning, USA). We added in the top well the necessary volume from the stock solution to reach $500 \mathrm{ppm}$ concentration of the NPs in a final volume of $200 \mu \mathrm{L}$. Then, serial dilutions were performed by taking $100 \mu \mathrm{L}$ from every very next well into $100 \mu \mathrm{L}$ of culture media. The last $100 \mu \mathrm{L}$ from the last dilution was discarded. The concentration range was from 250 to $0.97 \mathrm{ppm}$ of each NPs after the bacteria was added. For inoculation, an overnight (ON) culture was prepared at $37^{\circ} \mathrm{C}, 150 \mathrm{rpm}$, and incubated for $20 \mathrm{~h}$, Next, the ON culture was diluted 1:250 in fresh media and incubated until a critical optical density was reached $\left(\mathrm{OD}_{600 \mathrm{~nm}}\right.$ of $0.2 \pm 0.02$ for bacteria and an $\mathrm{OD}_{600 \mathrm{~nm}}$ of $0.3 \pm 0.02$ cells $/ \mathrm{mL}$ for yeast). Finally, a 1:100 dilution was made with fresh media, and $100 \mu \mathrm{L}$ of this dilution was added to each test well. Plates were incubated at $37^{\circ} \mathrm{C} 150 \mathrm{rpm}$ for $20 \mathrm{~h}$, and then, the $\mathrm{OD}_{600 \mathrm{~nm}}$ was measured in a Multiskan GO Microplate Spectrophotometer (Thermo Fisher Scientific, USA). The MIC was defined as the minimal concentration at which no growth was observed. Each assay was performed in triplicates; growth and sterility controls were included.

The behavior of the bimetallic NPs against microbial growth was analyzed by the Bliss independence model as described by Hegreness et al. ${ }^{56}$, which indicates that synergy can be considered when the effect of the combined antimicrobial agent (bimetallic NP) is more significant than the predicted of its components (single metal NP). Therefore, Eq. 1 was used to analyze the synergistic properties of the bimetallic nanoparticles, where the $S$ value; the difference between the predicted value of individual components $x$ and $y\left(f_{x 0}\right.$ and $f_{0 y}$, respectively) and the combined treatment $x y\left(f_{x y}\right)$ is denoted in the form:

$$
S=\left(\frac{f_{x 0}}{f_{00}}\right)\left(\frac{f_{0 y}}{f_{00}}\right)-\frac{f_{x y}}{f_{00}} \quad \text { Bliss Independence Model }
$$

where, $f_{x 0}=$ treatment with $\mathrm{Ag}, f_{0 y}=$ treatment with the transition metal, $f_{x y}=$ treatment $\mathrm{Ag}+$ metal, $f_{00}=$ growth control of a culture that has not been treated with the transition metals. The value of $S$ describes the interaction between combinatorial treatments as follows: $S>0$ Synergistic; $S=0$ Additive; $S<0$ Antagonistic.

Determination of minimum bactericidal and minimum fungicidal concentration (MBC, MFC). After obtaining the monometallic and bimetallic nanoparticles MICs, the wells that corresponded to the twice of the MIC, the MIC, and the growth control of each treatment were selected. Serial dilutions of selected wells were performed until they reach a final concentration of $1 \times 10^{7}$ cells $/ \mathrm{mL}$. Ten microliters of each dilution were cultured in a Petri dish, incubated at $37^{\circ} \mathrm{C}$ for $24 \mathrm{~h}$, and finally, counted the colony-forming units (CFU) in each treatment.

Received: 22 June 2020; Accepted: 16 February 2021

Published online: 05 March 2021

\section{References}

1. World Health Organization [WHO]. Antimicrobial resistance. WHO (2020). Available at: https://www.who.int/news-room/factsheets/detail/antimicrobial-resistance. Accessed: 10th Oct 2020

2. WHO. New report calls for urgent action to avert antimicrobial resistance crisis. Joint News Release 29, (2019).

3. Sánchez-López, E. et al. Metal-based nanoparticles as antimicrobial agents: an overview. Nanomaterials 10, 292 (2020).

4. Arora, N., Thangavelu, K. \& Karanikolos, G. N. Bimetallic nanoparticles for antimicrobial applications. Front. Chem. 8, 412 (2020).

5. Belenov, S. V. et al. Phase behavior of Pt-Cu nanoparticles with different architecture upon their thermal treatment. Nanotechnologies Russ. 12, 147-155 (2017)

6. Behera, A., Mittu, B., Padhi, S., Patra, N. \& Singh, J. Bimetallic nanoparticles: Green synthesis, applications, and future perspectives. In Multifunctional Hybrid Nanomaterials for Sustainable Agri-Food and Ecosystems 639-682 (Elsevier, 2020). doi:https://doi. org/10.1016/B978-0-12-821354-4.00025-X 
7. Sharma, G. et al. Novel development of nanoparticles to bimetallic nanoparticles and their composites: a review. J. King Saud Univ. - Sci. https://doi.org/10.1016/J.JKSUS.2017.06.012 (2017).

8. Kuppusamy, P., Yusoff, M. M., Maniam, G. P. \& Govindan, N. Biosynthesis of metallic nanoparticles using plant derivatives and their new avenues in pharmacological applications: an updated report. Saudi Pharm. J. 24, 473-484 (2016).

9. Mohamad, N. A. N., Jai, J., Arham, N. A. \& Hadi, A. A short review on the synthesis of bimetallic nanoparticles using plant extract. In: 2013 IEEE International Conference on Control System, Computing and Engineering 334-339 (IEEE, 2013). doi:https://doi. org/10.1109/ICCSCE.2013.6719985

10. Xiao, W., Li, S., Wang, S. \& Ho, C.-T. Chemistry and bioactivity of Gardenia jasminoides. J. Food Drug Anal. 25, 43-61 (2017).

11. Kesavan, K., Gnanasekaran, J., Gurunagarajan, S. \& Nayagam, A. A. J. microscopic, physicochemical and phytochemical analysis of gardenia jasminoides (ellis). Int. J. Pharm. Pharm. Sci. 10, 97 (2018).

12. Lü, F., Gao, Y., Huang, J., Sun, D. \& Li, Q. Roles of biomolecules in the biosynthesis of silver nanoparticles: case of gardenia jasminoides extract. Chin J. Chem. Eng. 22, 706-712 (2014).

13. Naseem, T. \& Farrukh, M. A. Antibacterial activity of green synthesis of iron nanoparticles using Lawsonia inermis and Gardenia jasminoides leaves extract. J. Chem. 2015, 1-7 (2015).

14. Jia, L., Zhang, Q., Li, Q. \& Song, H. The biosynthesis of palladium nanoparticles by antioxidants in Gardenia jasminoides Ellis: long lifetime nanocatalysts for p-nitrotoluene hydrogenation. Nanotechnology 20, 385601 (2009).

15. Meena Kumari, M., Jacob, J., Philip, D. Green synthesis and applications of Au-Ag bimetallic nanoparticles. Spectrochim. Acta Part A Mol. Biomol. Spectrosc. 137, 185-192 (2015).

16. Li, J. et al. Biosynthesis of $\mathrm{Au}, \mathrm{Ag}$ and $\mathrm{Au}-\mathrm{Ag}$ bimetallic nanoparticles using protein extracts of Deinococcus radiodurans and evaluation of their cytotoxicity. Int. J. Nanomedicine 13, 1411-1424 (2018).

17. Chavez, K. \& Rosas, G. Green synthesis and characterization of Ag@Au core-shell bimetallic nanoparticles using the extract of hamelia patens plant. Microsc. Microanal. 25, 1102-1103 (2019).

18. Botha, T. L. et al. Cytotoxicity of Ag, Au and Ag-Au bimetallic nanoparticles prepared using golden rod (Solidago canadensis) plant extract. Sci. Rep. 9, 4169 (2019).

19. Sharma, V. K., Siskova, K. M. \& Zboril, R. Magnetic Bimetallic Fe/Ag Nanoparticles: Decontamination and Antimicrobial Agents. In: ACS Symposium Series 1150, 193-209 (American Chemical Society, 2013).

20. Gallo, A., Bianco, C., Tosco, T., Tiraferri, A. \& Sethi, R. Synthesis of eco-compatible bimetallic silver/iron nanoparticles for water remediation and reactivity assessment on bromophenol blue. J. Clean. Prod. 211, 1367-1374 (2019).

21. Al-Asfar, A., Zaheer, Z. \& Aazam, E. S. Eco-friendly green synthesis of Ag@Fe bimetallic nanoparticles: antioxidant, antimicrobial and photocatalytic degradation of bromothymol blue. J. Photochem. Photobiol. B Biol. 185, 143-152 (2018).

22. Cusimano, M. G. et al. Biogenic iron-silver nanoparticles inhibit bacterial biofilm formation due to Ag+ release as determined by a novel phycoerythrin-based assay. Appl. Microbiol. Biotechnol. 104, 6325-6336 (2020).

23. Medina-Cruz, D. et al. Bimetallic Nanoparticles for Biomedical Applications: A Review. in Racing for the Surface 397-434 (Springer International Publishing, 2020). doi:https://doi.org/10.1007/978-3-030-34471-9_16

24. Zhang, X. F., Liu, Z. G., Shen, W. \& Gurunathan, S. Silver nanoparticles: Synthesis, characterization, properties, applications, and therapeutic approaches. Int. J. Mol. Sci. 17, (2016).

25. Klačanová, K. et al. Formation of $\mathrm{Fe}(0)$-nanoparticles via reduction of $\mathrm{Fe}(\mathrm{II})$ compounds by amino acids and their subsequent oxidation to iron oxides. J. Chem. 2013, 1-10 (2013).

26. Fakhri, A., Tahami, S. \& Naji, M. Synthesis and characterization of core-shell bimetallic nanoparticles for synergistic antimicrobial effect studies in combination with doxycycline on burn specific pathogens. J. Photochem. Photobiol. B Biol. 169, 21-26 (2017).

27. Gopinath, K. et al. Green synthesis of silver, gold and silver/gold bimetallic nanoparticles using the Gloriosa superba leaf extract and their antibacterial and antibiofilm activities. Microb. Pathog. 101, 1-11 (2016).

28. Uddin, R. et al. HPLC-analysis of polyphenolic compounds in gardenia jasminoides and determination of antioxidant activity by using free radical scavenging assays. Adv. Pharm. Bull. 4, 273-281 (2014).

29. Pierna, J. A. F. et al. Characterization and discrimination of phenolic compounds using Fourier transform Raman spectroscopy and chemometric tools. https://popups.uliege.be:443/1780-4507 (2018). doi:https://doi.org/10.25518/1780-4507.16270

30. Ruíz-Baltazar, A. Structural characterization of Fe-Ag bimetallic nanoparticles synthesized by chemical reduction. Int. Res. J. Pure Appl. Chem. 4, 263-269 (2014).

31. Gong, P. et al. Preparation and antibacterial activity of $\mathrm{Fe}_{3} \mathrm{O}_{4} @ \mathrm{Ag}$ nanoparticles. Nanotechnology 18, 285604 (2007).

32. Paszkiewicz, M. et al. Synthesis and characterization of monometallic ( $\mathrm{Ag}, \mathrm{Cu}$ ) and bimetallic $\mathrm{Ag}-\mathrm{Cu}$ particles for antibacterial and antifungal applications. J. Nanomater. 2016, 1-11 (2016).

33. Carroll, K. J. et al. One-pot aqueous synthesis of Fe and Ag core/shell nanoparticles. Chem. Mater. 22, 6291-6296 (2010).

34. Singh, P., Kim, Y. J., Wang, C., Mathiyalagan, R. \& Yang, D. C. The development of a green approach for the biosynthesis of silver and gold nanoparticles by using Panax ginseng root extract, and their biological applications. Artif. Cells Nanomed. Biotechnol. 44, 1-8 (2015).

35. Prabhu, Y. T., Rao, K. V., Kumari, B. S., Kumar, V. S. S. \& Pavani, T. Synthesis of $\mathrm{Fe}_{3} \mathrm{O}_{4}$ nanoparticles and its antibacterial application. Int. Nano Lett. 5, 85-92 (2015).

36. Guo, T. et al. The recent advances of magnetic nanoparticles in medicine. J. Nanomater. 2018, 1-8 (2018).

37. Srinoi, P., Chen, Y.-T., Vittur, V., Marquez, M. \& Lee, T. Bimetallic nanoparticles: enhanced magnetic and optical properties for emerging biological applications. Appl. Sci. 8, 1106 (2018).

38. Aslam, B. et al. Antibiotic resistance: a rundown of a global crisis. Infect. Drug Resist. 11, 1645-1658 (2018).

39. Morones, J. R. et al. The bactericidal effect of silver nanoparticles. Nanotechnology 16, 2346-2353 (2005).

40. Morones-Ramirez, J. R., Winkler, J. A., Spina, C. S. \& Collins, J. J. Silver Enhances antibiotic activity against gram-negative bacteria. Sci. Transl. Med. 5, 190ra81-190ra81 (2013).

41. Jalal, M. et al. Anticandidal activity of biosynthesized silver nanoparticles: effect on growth, cell morphology, and key virulence attributes of Candida species. Int. J. Nanomed. 14, 4667-4679 (2019).

42. Saqib, S. et al. Synthesis, characterization and use of iron oxide nano particles for antibacterial activity. Microsc. Res. Tech. 82, $415-420$ (2019).

43. Vitta, Y., Figueroa, M., Calderon, M. \& Ciangherotti, C. Synthesis of iron nanoparticles from aqueous extract of Eucalyptus robusta Sm and evaluation of antioxidant and antimicrobial activity. Mater. Sci. Energy Technol. 3, 97-103 (2020).

44. Chudasama, B., Vala, A. K., Andhariya, N., Upadhyay, R. V. \& Mehta, R. V. Enhanced antibacterial activity of bifunctional $\mathrm{Fe}_{3} \mathrm{O}_{4}-\mathrm{Ag}$ Core-Shell nanostructures. Nano Res 2, 955-965 (2009).

45. Marková, Z. et al. Air stable magnetic bimetallic Fe-Ag nanoparticles for advanced antimicrobial treatment and phosphorus removal. Environ. Sci. Technol. 47, 5285-5293 (2013).

46. Pillai, S. K., Moellering, R. C. \& Eliopoulos, G. M. Antimicrobial combinations. . Antibiot Lab. Med. 5, 365-440 (2005).

47. Garza-Cervantes, J. A. et al. Re-sensitizing ampicillin and kanamycin-resistant E. coli and S. aureus using synergistic metal micronutrients-antibiotic combinations. Front. Bioeng. Biotechnol. 8, 612 (2020).

48. Roy, N., Gaur, A., Jain, A., Bhattacharya, S. \& Rani, V. Green synthesis of silver nanoparticles: an approach to overcome toxicity. Environ. Toxicol. Pharmacol. https://doi.org/10.1016/j.etap.2013.07.005 (2013).

49. Neal, A. L. What can be inferred from bacterium-nanoparticle interactions about the potential consequences of environmental exposure to nanoparticles?. Ecotoxicology 17, 362-371 (2008). 
50. Prabhu, S. \& Poulose, E. K. Silver nanoparticles: mechanism of antimicrobial action, synthesis, medical applications, and toxicity effects. Int. Nano Lett. 2, 32 (2012).

51. Pankey, G. A. \& Sabath, L. D. Clinical relevance of bacteriostatic versus bactericidal mechanisms of action in the treatment of gram-positive bacterial infections. Clin. Infect. Dis. 38, 864-870 (2004).

52. Finberg, R. W. et al. The importance of bactericidal drugs: future directions in infectious disease. Clin. Infect. Dis. 39, 1314-1320 (2004).

53. de Oliveira Gonçalves, K., Vieira, D. P., Levy, D., Bydlowski, S. P. \& Courrol, L. C. Uptake of silver, gold, and hybrids silver-iron, gold-iron and silver-gold aminolevulinic acid nanoparticles by MCF-7 breast cancer cells. Photodiagnosis Photodyn. Ther. https:// doi.org/10.1016/j.pdpdt.2020.102080 (2020).

54. Ramírez-Acosta, C. M., Cifuentes, J., Cruz, J. C. \& Reyes, L. H. Patchy core/shell, magnetite/silver nanoparticles via green and facile synthesis: routes to assure biocompatibility. Nanomaterials 10, 1857 (2020).

55. Kaur, P., Thakur, R., Malwal, H., Manuja, A. \& Chaudhury, A. Biosynthesis of biocompatible and recyclable silver/iron and gold/ iron core-shell nanoparticles for water purification technology. Biocatal. Agric. Biotechnol. 14, 189-197 (2018).

56. Hegreness, M., Shoresh, N., Damian, D., Hartl, D. \& Kishony, R. Accelerated evolution of resistance in multidrug environments. 105, 13977-13981 (2008)

\section{Acknowledgements}

The authors want to thank to the Universidad Autonoma de Nuevo León and CONACyT for providing financial support through Paicyt 2016-2017, Paicyt 2019-2020 and Paicyt 2020-2021 Science Grants. CONACyT Grants for: Basic science grant 221332, Fronteras de la Ciencia grant 1502 and Infraestructura Grant 279957. Ana L Padilla Cruz for the support from a Beca Nacional de Posgrado from CONACyT and Dr. Angel Leon Buitimea for the support from a Beca de Posdoctorado Nacional. We also want to thank to the Universidad Autonoma de Nuevo Leon and the Centro de Investigación en Biotecnología y Nanotecnología for providing the infrastructure and equipment to perform the experiments.

\section{Author contributions}

Conceptualization, A.L.B., and J.R.M.R. Writing-original draft preparation A.L.P.C., J.A.G.C., X.G.V.A., A.L.B. and J.R.M.R., writing-review, and editing, A.L.P.C., J.A.G.C., G.G.R., A.L.B. and J.R.M.R. Supervision, A.L.B., and J.M.R. All authors reviewed the final version of the article and approved the submitted version.

\section{Competing interests}

The authors declare no competing interests.

\section{Additional information}

Correspondence and requests for materials should be addressed to J.R.M.-R.

Reprints and permissions information is available at www.nature.com/reprints.

Publisher's note Springer Nature remains neutral with regard to jurisdictional claims in published maps and institutional affiliations.

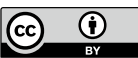

Open Access This article is licensed under a Creative Commons Attribution 4.0 International License, which permits use, sharing, adaptation, distribution and reproduction in any medium or format, as long as you give appropriate credit to the original author(s) and the source, provide a link to the Creative Commons licence, and indicate if changes were made. The images or other third party material in this article are included in the article's Creative Commons licence, unless indicated otherwise in a credit line to the material. If material is not included in the article's Creative Commons licence and your intended use is not permitted by statutory regulation or exceeds the permitted use, you will need to obtain permission directly from the copyright holder. To view a copy of this licence, visit http://creativecommons.org/licenses/by/4.0/.

(C) The Author(s) 2021 